\title{
Whole-Genome and Transcriptome Sequencing Identified NOTCH2 and HES1 As Potential Markers of Response to Imatinib in Desmoid Tumor (Aggressive Fibromatosis): A Phase II Trial Study
}

Joonha Kwon

Korea Advanced Institute of Science and Technology

Jun Hyeong Lee

Korea Advanced Institute of Science and Technology

Young Han Lee

Yonsei University College of Medicine

Jeeyun Lee

Samsung Medical Center

Jin-Hee Ahn

Asan Medical Center

Se Hyun Kim

Seoul National University Bundang Hospital

Seung Hyun Kim

Yonsei University College of Medicine

Tae II Kim

Yonsei University College of Medicine

Kum-Hee Yun

Yonsei University College of Medicine

Young Suk Park

Samsung Medical Center

Jeong Eun Kim

Asan Medical Center

Kyu Sang Lee

Seoul National University Bundang Hospital

Jung Kyoon Choi

Korea Advanced Institute of Science and Technology

Hyo Song Kim ( $\sim$ hyosong77@yuhs.ac)

Yonsei University College of Medicine https://orcid.org/0000-0002-0625-9828 
Research

Keywords: Desmoid tumor, Imatinib, Phase II clinical trial, NOTCH2, HES1

Posted Date: October 26th, 2021

DOI: https://doi.org/10.21203/rs.3.rs-993539/v1

License: (c) (i) This work is licensed under a Creative Commons Attribution 4.0 International License. Read Full License 


\section{Abstract}

Background: Desmoid tumor, also known as aggressive fibromatosis, is well-characterized by abnormal Wnt/ $\beta$-catenin signaling. Various therapeutic options, including imatinib, are available to efficiently treat desmoid tumor. However, molecular mechanism of why imatinib works remains poorly understood. Here, we describe the potential roles of $\mathrm{NOTCH} 2$ and $\mathrm{HES} 1$ in association with clinical response to imatinib as in genome and transcriptome levels.

Methods: We identified all somatic mutations in coding and non-coding regions via whole genome sequencing using desmoid tumor samples. To validate the genetic interaction with expression level in desmoid-tumor condition, we utilized large-scale Whole-genome sequencing (WGS) and transcriptome datasets from the Pan-Cancer Analysis of Whole Genomes (PCAWG) project. RNA-sequencing was performed using prospective and retrospective cohort samples to evaluate the expressional relevance with clinical response.

Results: Among 20 patients, 4 (20\%) had a partial response and 14 (66.7\%) had stable disease, 11 of which continued for $\geq 1$ year. With gene-wise functional analyses, we detected significant correlation between recurrent $\mathrm{NOTCH} 2$ noncoding mutations and clinical response to imatinib. Based on PCAWG data analyses, NOTCH2 mutations affect its expression levels particularly in the presence of CTNNB1 missense mutations. By analyzing RNA-sequencing with additional desmoid tumor samples, we found that NOTCH2 expression was significantly correlated with HES1 expression. Interestingly, NOTCH2 had no statistical power to discriminate responders and non-responders. Instead, HES1 was differentially expressed with statistical significance between responders and non-responders

Conclusions: Imatinib was effective and well tolerated for advanced desmoid tumor treatment. Our results show that $H E S 1$, regulated by NOTCH2, as an indicator of sensitivity to imatinib, which provides an important therapeutic consideration for desmoid tumor.

Trial Registration: ClinicalTrials.gov, NCT02495519, Registered July 13, 2015- Retrospectively registered, https://www.clinicaltrials.gov/ct2/show/NCT02495519

\section{Background}

A desmoid tumor (aggressive fibromatosis) is fibroproliferative neoplasm arising from deep connective tissues. A stepwise approach including an active surveillance is established as no metastatic potential and spontaneous tumor regression are observed[1]. Excision is the mainstay of treatment, but the postsurgical recurrence rate is high [2,3]. For unresectable or recurrent desmoid tumors, a variety of systemic therapeutic options are available, including tamoxifen, nonsteroidal anti-inflammatory drugs (NSAIDs), interferons, and chemotherapy [4-8]. Recently, sorafenib induced durable response and led to its approval for the disease treatment [9]. Imatinib is a new treatment option in unresectable, progressive, or recurrent desmoid tumors [10-12]. In addition to its promising efficacy (6-15\% response rate), the favorable toxicity profiles support its long-term use for salvage treatment. In imatinib-responsive 
diseases, such as gastrointestinal stromal tumors or chronic myeloid leukemia, specific mutations or chromosomal translocations have been reported [13-15]. However, in desmoid tumors, no molecular abnormalities in imatinib-sensitive kinases have been observed $[10,11]$. Therefore, molecular mechanisms by which this rare tumor responds to imatinib are poorly understood.

In this study, we conducted a multicenter phase II trial to evaluate the efficacy of imatinib in patients with relapsing or progressive desmoid tumors. To understand the molecular basis of the clinical responses to imatinib, we performed whole-genome sequencing to identify potential markers. Owing to limited insights gained from protein coding mutations, we extended our analyses to noncoding regulatory regions. Our gene-wise recurrence model using 1,009 pan-cancer whole-genome data indicated that NOTCH2 regulatory mutations are associated with the response of desmoid tumors to imatinib. We further evaluated the significance of $\mathrm{NOTCH} 2$ in transcriptome analysis using RNA-sequencing data. We discovered that HES1, a well-known downstream target of Notch signaling pathway, is directly associated with imatinib sensitivity.

\section{Methods}

\section{Patients and Treatment}

Patients with advanced desmoid tumors, defined as patients with radiographic progression after previous treatment, were eligible for prospective phase II study. Key inclusion criteria were as follows: age $\geq 10$ years, Eastern Cooperative Oncology Group performance status of 0 to 2, and adequate hematologic and renal function. Patients were treated with $400 \mathrm{mg}$ of imatinib mesylate (Glima; Boryung Pharmaceutical Co., Ltd, Seoul, Korea) daily until progression or unacceptable toxicity. Toxic effects were graded according to the National Cancer Institute-Common Toxicity Criteria v. 4.03. Disease was assessed every 8 weeks for the initial 32 weeks and then every 16 weeks according to RECIST (response evaluation criteria in solid tumors) v1.1 [16]. Briefly, patients who experienced grade $3 / 4$ toxicity or intolerable grade 2 toxicity stopped treatment and then restarted at a reduced dose (300 mg/day or $200 \mathrm{mg} /$ day). The study was approved by the institutional review board (IRB No. 2013-1417-001).

Retrospective cohort study included 24 additional patients with desmoid tumor and approved by the institutional review board (IRB No. 2020-2244-001). Surgically resected formalin-fixed paraffin-embedded (FFPE) tissue samples obtained prior to radiotherapy or chemotherapy were subjected to transcriptome sequencing (Fig. 1). Of those, 4 cases were treated with imatinib and remaining 20 were treatment naïve.

\section{DNA Extraction and Quality Assessment}

Whole genome sequencing was performed using pretreatment tumor excision samples as well as matched blood samples. Briefly, 4-mm-thick sections with a tumor content of $\geq 80 \%$ were obtained, and $\geq 2 \mu \mathrm{g}$ of DNA was extracted using the Maxwell 16 FFPE Plus LEV DNA Purification Kit (Promega, Madison, WI, USA). For peripheral blood mononuclear cells (PBMCs), genomic DNA was extracted using the QIAamp DNA Mini Kit (Qiagen, Hilden, Germany) as per the manufacturer's instructions. 
Library preparation was performed using the TruSeq Nano DNA Library Preparation Kit (Illumina, San Diego, CA, USA) following the manufacturer's instructions. Illumina utilizes a unique "bridged" amplification reaction on the surface of the flow cell. A flow cell containing libraries was prepared using the cBot Fluidics Station and was then loaded into the HiSeq X-10 sequencer (Illumina) for automated cycles of extension and imaging. Sequencing-by-Synthesis cycles were repeated to achieve a paired-end read length of $2 \times 150 \mathrm{bp}$.

\section{RNA Library Construction and Whole Transcriptome Sequencing}

Total RNAs were extracted and purified from frozen tumor samples with ReliaPrep FFPE Total RNA Miniprep System (Promega, Inc.) according to the manufacturer's procedures. Amount of RNA and its quality were checked on an Agilent RNA 6000 Nano Kit (Agilent Technologies). For analysis of RNAsequencing data, we prepared mRNA sequencing libraries as paired-end reads with a length of 100 bases using the SMARTer Stranded Total RNA-seq kit v2-Pico Input Mammalian according to the manufacturers' protocols. Briefly, mRNA molecules were purified and fragmented from $2 \mu \mathrm{g}$ of total RNA. The libraries were sequenced as paired end reads ( $2 \times 150$ bp) using the NovaSeq 6000 (Illumina).

\section{Whole Genome Data Processing}

To process whole genome sequencing data of desmoid tumors, we adopted the Genome Analysis Tool Kit (GATK) v3.7 best practice provided by the Broad Institute [17]. Briefly, we mapped qualified paired-end reads to the human reference genome (hg19) with Burrows-Wheeler Aligner (BWA) 0.7.15 [18]. Subsequently, we filtered PCR duplicates using Picard tools 2.8.2 to remove potential bias that occurred during sequencing processes. Then, we performed recommended procedures, such as local realignment and base quality recalibration to extract analysis-ready reads.

\section{Somatic Variant Detection}

MuTect2 [19] of the GATK pipeline with default parameters was used to identify somatic single nucleotide variants (SNVs) and small insertions/deletions (indels). The processed whole-genome-sequencing data for tumor and matched normal samples (PBMCs) were used in BAM format as inputs for Mutect2 [19]. Somatic variants were annotated using ANNOVAR [20]. Some candidate variants were manually inspected using Integrative Genomics Viewer (IGV) [21]. Population-level allele frequencies of candidates were obtained using Genome Aggregation Database (gnomAD) [22]. For two samples with tumor data only, normal sample data for one of the nine other patients was used as matched normal control for variant calling. A sample that was sequenced in the same batch with a read depth of greater than 30 was used. The variants were further filtered using gnomAD to obtain putative somatic mutations in the tumoronly samples.

\section{Scoring Gene-Wise Recurrence of Functional Variants}


Our previously developed gene-wise recurrence model was used [23]. Conventionally, mutations are considered recurrent if and only if they occur at the same genomic location across multiple samples. Mutations are considered oncogenic when their recurrence exceeds a certain threshold [24]. However, this definition of recurrence is inappropriate for analyses of noncoding regions owing to their vast size. Thus, we consider mutations recurrent if they occur in functional regions of the same gene, even if they are not recurrent in a site-specific manner. In particular, we focused on mutational events in cis-regulatory regions of a mammalian gene dispersed across a long range in the genome [25]. Genes were defined based on the GENCODE v.19 gene set mapped to GRCh37 [26].

To identify coding and noncoding mutations with significant functional consequences, deleterious effects of each SNV were predicted using two algorithms, Combined Annotation-Dependent Depletion (CADD) [27] and Deleterious Annotation of genetic variants using Neural Networks (DANN) [28]. Both models were trained to distinguish benign variants from deleterious variants $[27,28]$. For multiple mutations in the same gene, the one with the highest score for deleteriousness was selected to represent the functional consequence.

\section{Reference Whole-Genome and Transcriptome Datasets}

To characterize the functional effects of $\mathrm{NOTCH} 2$ noncoding mutations, a large-scale pan-cancer dataset consisting of somatic variants from whole-genome sequencing data and transcriptome data for tumor and matched normal samples were used. VCF files for somatic variant calling and gene expression matrices containing FPKM-upper quantile values were obtained from the Pan-Cancer Analysis of Whole Genomes (PCAWG) Project[29].

\section{RNA-sequencing data processing and QC}

We generated RNA-sequencing data of 31 desmoid tumor patients. We removed adapter sequences using Cutadpt [30], and aligned the trimmed reads using STAR [31] with hg19. Gene expression was quantified using RSEM [32]. Quality control check at pre-alignment step was conducted using FASTQC and at postalignment step using RSeQC [33]. QC results were visualized with MultiQC [34]. At post-alignment step, we noticed two patients with potential problems in read distribution, and infer experiment criteria. Thus, we excluded those samples from future analysis.

\section{Bioinformatics and Statistical Analyses}

The $\chi^{2}$ test was used to assess correlations between marker status and clinical significance. All correlation analysis was conducted using spearman correlation. To assess statistical significance between responders and non-responders of imatinib, we calculated Mann-Whitney $U$ test. All tests were two-sided and $\mathrm{P}<0.05$ is considered significant. Cleveland, scatter and box plots were generated by using ggplot2 $\mathrm{R}$ package and matplotlib python package. 
To conduct enrichment analysis, we adopted two approaches. First of all, we identified genes that are significantly correlated with imatinib sensitivity and used those genes as input for EnrichR [35]. As an alternative step, we conducted Gene Set Enrichment Analysis (GSEA) between responders and nonresponders using C2, C5, C6 and Hallmark MSigDB gene sets [36]. C5 Notch category was defined as Notch-related terms present in $\mathrm{C} 5$ category.

Progression-free survival (PFS) was calculated from start date of imatinib to date of progression or death and progression free rate at 16 weeks (PFR 16) was defined as proportions of patients without progression at 16 weeks, analyzed using the Kaplan-Meier method [SPSS version 18.0 (IBM, Chicago, IL, USA)].

\section{Results}

\section{Sample Set, Patient Outcomes and Toxicity}

Total of 21 patients were enrolled between April 2014 and October 2015. One patient withdrew, leaving 20 patients (Fig. 1; Additional file 1: Table S1). Three patients (7, 8, and 13) had a known diagnosis of familial adenomatous polyposis (FAP). Fifteen patients (75\%) underwent one previous surgery. Most of the patients had been treated with non-surgical procedures, including radiotherapy $(6,30.0 \%)$ and/or chemotherapy $(12,60.0 \%)$.

Of the 20 evaluable patients, $4(20 \%)$ had partial responses (PR) to treatment with durations of 6.7, 26.8 (Fig. 2A), 30.3, and 35.1 (Fig. 2B) months. One PR case had FAP. The PR duration was longer than 1 year for three patients. Fig. $2 \mathrm{C}$ provides a waterfall plot of the best response; 14 patients $(66.7 \%)$ had stable disease (SD), and the clinical benefit rate was $90.0 \%$. The median time to progression was 21.4 months (range: 2.8 to 40.7 months) and PFR 16 was $85 \%$ (Fig. 2D).

In terms of toxicity, $400 \mathrm{mg}$ of imatinib was well tolerated with expected grade $3 / 4$ toxicities: neutropenia $(n=1)$, anorexia $(n=1)$, vomiting $(n=1)$, and fatigue $(n=1)$ (Additional file 2: Table S2). Three patients had a one dose level reduction $(300 \mathrm{mg} / \mathrm{d})$, and one patient had a reduction of two levels $(200 \mathrm{mg} / \mathrm{d})$ owing to toxicity.

\section{Results of Whole-Genome Sequencing}

After confirming adequate DNA quantities, whole genome sequencing was performed for 11 samples. Two samples (patients 11 and 13) without matched control data were excluded from the primary analysis and used for extensional validation only (Fig. 1). The average read depth was greater than 21.46 (range: 21.46 to 54.05) (Additional file 3: Table S3). By implementing the GATK pipeline from the Broad Institute, we identified 832-4,110 SNVs and indels per sample and used ANNOVAR for annotation (Additional file 4: Table S4).

Next, we examined mutational signatures from annotated variants to identify types of mutational processes [37]. Signature $1 \mathrm{~A}$ was dominant, accounting for around $88 \%$ of signatures (Additional file 5 : 
Table S5), indicating that desmoid tumor variants primarily arise due to errors in replicative polymerases in the DNA repair pathway [37]. The majority of mutations were in intronic regions. Mutations in coding regions were primarily missense and synonymous variants (Additional file 6: Fig. S1).

We also investigated alterations of cancer driver genes registered in the COSMIC database [38] and detected $C T N N B 1$ mutations in 8 out of 11 samples, while 2 patients harbored adenomatous polyposis coli $(A P C)$ mutations. CTNNB1 and APC mutation sites of patients are summarized in Fig. 2C. All mutations affected phosphorylation sites necessary for the proper degradation of $\beta$-catenin [39-41]. Remarkably, no other mutations in COSMIC cancer driver genes were detected, emphasizing the prominent role of the Wnt/ $\beta$-catenin signaling pathway in desmoid tumor progression [8].

\section{Gene-Wise Recurrence Analysis of Potential Cancer- Associated Genes}

To identify potential cancer-associated mutations in desmoid tumors, we employed gene-wise recurrence analysis of mutations in noncoding regions, according to previously developed method [23]. Briefly, the model assumes that mutations in multiple patients are recurrent if they affect the same gene (Fig. 3A). Recurrently affected genes were defined as those with mutations in at least two samples. This strategy allowed us to evaluate noncoding regions with potentially significant impacts on gene regulation with limited sample size. NOTCH2, RGPD2, and ARHGAP11B were identified as strong candidates (Fig. 3B).

\section{Correlation between Gene-Wise Scores and Imatinib Sensitivity}

We examined the association between the change in tumor volume after imatinib treatment and the deleteriousness score of recurrently mutated genes. In case of $\mathrm{NOTCH}$, tumor volume changes were highly correlated with both CADD $(R=-0.797, P=0.01)$ and DANN scores $(R=-0.831, P=0.006)$ (Fig. 3B and C; Additional file 7: Table S6). This correlation was maintained even when two tumor-only samples (patient 11 and 13) were included (Fig. 3C). According to the annotated information of somatic variants in NOTCH2, three (patients 3,5 , and 8 ) and four (patients $9,11,13$, and 18) variants were intergenic and intronic, respectively, while only one (patient 19) variant was nonsynonymous (Additional file 8: Table S7). Using gnomAD database, we found that the allele frequencies of the $\mathrm{NOTCH} 2$ variants were extremely low $(0-1.13 \%)$, indicating that they were likely somatic. These results suggest that the regulation of $\mathrm{NOTCH} 2$ at the gene level may contribute to the desmoid tumor response to imatinib.

\section{Regulatory Role of NOTCH2 Mutations}

To evaluate the regulatory effects of $\mathrm{NOTCH} 2$ mutations, we interrogated RNA sequencing and somatic mutation profiles from PCAWG datasets $(n=1,009)$. For comparison across cancer types, we transformed the NOTCH2 expression levels to Z-scores within each cancer type and identified samples with NOTCH2 mutations. Considering the role of CTNNB1 missense mutations in desmoid tumors [8], we further selected both-NOTCH2-CTNNB1 mutants in the PCAWG data (Fig. 4A; Additional file 9: Table S8). 
NOTCH2 expression levels were more highly correlated with CADD scores for both-NOTCH2-CTNNB1 mutation group $(R=0.607, P=0.013)$ than for $N O T C H 2$ mutation-only group $(R=0.178, P=0.01)$ (Fig.

$4 \mathrm{~B})$. The same trends were observed when DANN score was used for variant scoring ( $R=0.467)$ (Fig. 4C), although the correlation was only marginally significant $(P=0.068)$. These results suggest that genetic interactions between $N O T C H 2$ noncoding mutations and $C T N N B 1$ missense mutations may influence the NOTCH2 expression level.

\section{Role of NOTCH family members and HES1 in imatinib sensitivity}

To validate significance of our finding, we analyzed RNA-sequencing data of 29 desmoid tumor patients (Fig. 1; Additional file 10: Table S9). We focused on Notch family members, including NOTCH2, and HES1, a marker of stemness [42] that has been implicated as a target of Notch signaling pathway [43] and marker of imatinib sensitivity [44]. We first investigated whether expression levels of Notch family members and HES1 are correlated. We calculated spearman correlation between the genes, and discovered that $\mathrm{NOTCH} 2$ expression was significantly correlated with HES1 expression $(P=0.0091568)$ while expressions of other Notch genes were not (Fig. 5A). This recapitulates the association between HES1 and NOTCH2 detected in WGS analysis. We also noticed that all Notch family members are significantly correlated with each other (Additional file 11: Fig. S2). This suggests that other Notch family members can potentially participate in regulation of $H E S 1$ expression although $\mathrm{NOTCH} 2$ is most directly associated with HES1.

Next, we examined whether expression of Notch family members or HES1 is associated with imatinib response. We classified patients into responders $(P R, n=5)$ or non-responders $(S D / P D, n=6)$ on the basis of clinical implementation, and compared expression values between two groups. Strikingly, we found that none of Notch family members had statistical power to distinguish two groups. Intriguingly, HES1 was significant in discriminating patients' response $(P=0.027617)$ (Fig. 5B). This is in accordance with report that overexpression of HES1 sensitizes cells to imatinib in chronic myeloid leukemia model [44]. Furthermore, we calculated spearman correlation between response rate and expression levels. Initially, we found no correlation between HES1 or Notch family genes with tumor volume change, with marginal significance in $H E S 1(P=0.07)$. However, after removing outlier (defined as patient with highest gene expression), we found that $H E S 1$ was significantly correlated with clinical response $(P=0.00922195)$ (Fig. 5C). Still, none of Notch members, except NOTCH4, achieved statistical significance. Overall, HES1 was the most significant marker of imatinib sensitivity.

\section{Enrichment analysis of response-associated genes}

To identify biological pathways that are associated with imatinib sensitivity, we first conducted gene set enrichment analysis (GSEA) between responders and non-responders (Fig. 6A). Differentially expressed genes were enriched in diverse biological pathways including muscle cell cellular homeostasis, negative regulation of myoblast differentiation, and skeletal tissue regeneration, angiogenesis and regulation of oxidative phosphorylation. We also performed enrichment analysis using genes that are significantly 
correlated with imatinib sensitivity (Fig. 6B). Similar terms, such as fibroblast growth factor binding, muscle cell migration, and oxidative phosphorylation, were enriched. Among these terms, we found that glucose metabolism and mitochondrial respiration have been closely linked to imatinib sensitivity [45] and are upregulated in naïve pluripotent stem cells [46] in previous researches. In addition, angiogenesis is a well-known feature of mesenchymal stem cells [47]. Collectively, we propose that these terms, such as oxidative phosphorylation and angiogenesis, and HES1 all point to the significance of mesenchymal stem cell population that are prone to imatinib in desmoid tumor.

\section{Discussion}

Using whole-genome and transcriptome sequencing, we performed integrative molecular characterization of desmoid tumor in patients receiving imatinib treatment. Our analyses suggest HES1 overexpression, potentially regulated by $\mathrm{NOTCH} 2$, can serve as a predictor of the clinical response to imatinib in desmoid tumor patients. To our knowledge, this is the first integrative study to characterize molecular determinants of the response to imatinib in desmoid tumor.

Our coding-region analyses recapitulated previous findings on the prevalence of CTNNB1 and APC mutations [48]. Unfortunately, these mutations were not associated with clinical responses to imatinib. However, we discovered that mutations in noncoding regulatory regions of $\mathrm{NOTCH} 2$ are positively correlated with the clinical response to imatinib. Moreover, our investigation of PCAWG samples revealed that noncoding mutations in NOTCH2 regions increase expression. Importantly, NOTCH2 expression was significantly correlated with $H E S 1$, and HES1 was indicative of imatinib sensitivity in our desmoid cohort. We noticed that removal of one outlier restored statistical significance when assessing correlation between HES1 and tumor volume change. Still, our correlation analysis suggests that HES1 expression is the major determinant of imatinib sensitivity. Other minor factors of imatinib sensitivity need to be determined to fully elucidate mechanism of imatinib in desmoid tumor. Taken together, we suggest that HES1 and NOTCH2 overexpression is a predictor of the anti-cancer effects of imatinib on desmoid tumors.

Our study is not the very first attempt to investigate the significance of Notch signaling in desmoid tumor $[43,49]$. Based on multiple evidences, small molecule inhibitors, such as $\gamma$-secretase inhibitor, siRNA, and monoclonal antibody against Notch pathway were designed to treat desmoid tumor [50]. Studies have confirmed that activation of $\mathrm{NOTCH} 2$ leads to overexpression of $\mathrm{HES} 1$ and are accompanied by proliferation, immature morphology and aggressiveness in acute kidney injury model [51] and hepatocellular carcinoma model [52]. Of note, Notch signaling and HES1 have been associated with response to imatinib in Chronic Myeloid Leukemia cells [44, 53], providing robust evidence for NotchHES1 axis in mechanism of imatinib in desmoid tumor. Surprisingly, however, no research reported its association with clinical response of imatinib in desmoid tumor until now.

The mechanism underlying the tumor response to imatinib is still not fully understood. In agreement with our findings, multiple lines of evidence support their significance in desmoid tumor. In a preclinical study, 
imatinib inhibits Notch signaling by increasing the proteosomal degradation of intracellular Notch. Furthermore, a primary effector of Notch signaling, HES1, decreased as the imatinib concentration increased [54]. In a neurodegenerative condition, Alzheimer's disease, imatinib results in the dosedependent inhibition of $\gamma$-secretase activity [55]. Similarly, NSAIDs, another standard treatment for desmoid tumors, alter $\gamma$-secretase activity [56]. Taken together, these findings support the predictive value of Notch and HES1 as therapeutic strategy.

The Notch pathway exhibits crosstalk with the Wnt signaling cascade [57] and is involved in the regulation of tumor microenvironments and the maintenance of cancer stem cells [58-60]. With NOTCH1 activation, desmoid tumors showed high expression levels of $\mathrm{NOTCH} 1$ and its downstream transcription factor HES1 [43], of which transcriptional activity is dependent on NOTCH2 [61]. Thus, targeting Ysecretase to prevent Notch cleavage has been suggested as a novel therapeutic approach $[62,63]$. A phase II trial of the $y$-secretase inhibitor PF-03084014 demonstrated a promising efficacy, with a response rate of $29 \%$ for patients with progressive desmoid tumors [63]. Furthermore, the efficacy of PF-03084014 is high in tumors with elevated expression of genes in the Notch and Wnt pathways [64].

We sought to explain the increased correlation between $\mathrm{NOTCH} 2$ mutation scores and $\mathrm{NOTCH} 2$ expression levels when considering the CTNNB1 mutation status. A previous study has shown that the TCF4/ $\beta$-catenin complex binds to the promoters of Notch signaling pathway genes, including NOTCH2 [65]. In the absence of the TCF4/ $\beta$-catenin complex, the transcription machinery cannot be assembled at regulatory regions and fails to induce $\mathrm{NOTCH} 2$ expression, thereby preventing $\mathrm{NOTCH} 2$ regulatory mutations from exerting effects. We speculate that the hyperactivity of the TCF $4 / \beta$-catenin complex induced by $C T N N B 1$ msissense mutations leads to the constitutive activation of Notch signaling. This allows NOTCH2 regulatory mutations to alter gene expression levels, leading to a high correlation between deleteriousness and transcript levels. Further investigations, including functional studies, are needed to validate the mode of action of imatinib.

As mentioned earlier, mutation status of driver genes failed to discriminate patients who will respond to imatinib. We overcame this hurdle with analysis of non-coding mutations, providing rationale to investigate Notch signaling and its downstream target HES1 to interpret molecular mechanism of imatinib. Thus, significance of non-coding mutation confers huge advantage to whole-genome sequencing data over whole-exome sequencing data. Despite such significance, we acknowledge several limitations of our study. First of all, we are aware that limited sample size hinders more comprehensive study of desmoid tumor. For example, although we were initially unable to obtain statistical significance for $\mathrm{NOTCH}_{2}$ in Fig. 5B (responder versus non-responder analysis), removal of outlier restored statistical significance (p-value from 0.2 to 0.07 ), proving that $\mathrm{NOTCH} 2$ is a biologically meaningful biomarker. Rarity of desmoid tumor obscured the statistical power, and increased sample size will endow power to rescue unrecognized candidates. Also, we admit lack of functional study. Collectively, multiple evidences point out to Notch-HES1 axis in various studies, its significance in response to imatinib, and role of HES1 in stemness. Thus, future work should validate both clinical and biological significance of HES1 in desmoid tumor to gain insight into this aggressive rare tumor.

Page 11/26 
In conclusion, using whole-genome sequencing with gene-wise recurrence model and transcriptome of desmoid tumor, we propose that overexpression of NOTCH2 and HES1 is the marker of sensitivity for the anti-cancer effects of imatinib on desmoid tumors. Our results suggest that HES1 should especially be considered in clinical settings when using imatinib to treat this rare and challenging disease.

\section{Conclusions}

Our results show that $H E S 1$, regulated by $N O T C H 2$, as an indicator of sensitivity to imatinib, which provides an important therapeutic consideration for desmoid tumor. Integrative multi-omics data of desmoid tumor with gene-wise recurrent model provided novel insight into previously inexplicable mechanism of imatinib. Thus, non-coding mutations and subsequent gene expression change must be considered in clinical setting for efficient treatment.

\section{Abbreviations}

APC: Adenomatous Polyposis Coli

BWA: Burrows-Wheeler Aligner

CADD: Combined-Annotation Dependent Depletion

COSMIC: Catalogue of Somatic Mutations in Cancer

DANN: Deleterious Annotation of Genetic Variants Using Neural Networks

FAP: Familial Adenomatous Polyposis

FFPE: Formalin-fixed paraffin-embedded

FPKM: Fragments Per Kilobase of transcript per Million mapped reads

GATK: Genome Analysis Toolkit

gnomAD: Genome Aggregation Database

GSEA: Gene Set Enrichment Analysis

IGV: Integrative Genomics Viewer

Indels: Insertions/deletions

NSAID: Nonsteroidal anti-inflammatory drugs

PBMC: Peripheral blood nuclear mononuclear cells 
PCAWG: Pan-cancer Analysis of Whole Genomes

PD: Progressive Diseases

PFR: Progression-Free Rate

PFS: Progression-Free Survival

PR: Partial Responses

QC: Quality Control

SD: Stable Diseases

SNV: Somatic nucleotide variant

WGS: Whole-Genome Sequencing

\section{Declarations}

Ethics approval and consent to participate: The study was approved by the institutional review board (IRB No. 2013-1417-001). Retrospective cohort study of 24 additional patients with desmoid tumor was approved by the institutional review board (IRB No. 2020-2244-001).

Consent to publish: Written informed consent for publication was provided by the participants

Availability of data and materials: Variant Call Format (VCF) of whole-genome sequenced patients, gene expression matrix of RNA-sequenced patients, and clinical information used in this study are available from the corresponding author on request. PCAWG data was obtained from https://dcc.icgc.org/pcawg/ as instructed [29].

Competing Interests: The authors declare no potential conflicts of interest.

Funding: This work was supported by a grant from the National Research Foundation of Korea (NRF) funded by the Korean government (MSIT) [2018R1A2B6003707 to H.S.K., NRF-2017M3A9A7050612 to J.K.C.]; and the Severance Hospital Research Fund for Clinical Excellence (SHRC) [C-2019-0015].

Authors' Contributions: H.S.K and J.K.C conceived and designed the experiments. J.H.K and J.H.L analyzed and interpreted the data. Y.H.L, J.Y.L, J.H.A, S.H.K, S.H.K, T.I.K, K.H.Y, Y.S.P, J.E.K and K.S.L conducted clinical trial, prepared sample and generated data used in this study. J.H.K. and J.H.L were responsible for drafting the manuscript. H.S.K and J.K.C obtained the funding. All authors read and approved the final manuscript.

Author information: J.K and J.H.L contributed equally to this work. 
Corresponding authors: Correspondence to Hyo Song Kim and Jung Kyoon Choi.

Acknowledgement: Not applicable

\section{References}

1. Colombo C, Miceli R, Le Péchoux C, Palassini E, Honoré C, Stacchiotti S, et al. Sporadic extra abdominal wall desmoid-type fibromatosis: surgical resection can be safely limited to a minority of patients. Eur J Cancer England. 2015;51:186-92.

2. Lev D, Kotilingam D, Wei C, Ballo MT, Zagars GK, Pisters PWT, et al. Optimizing treatment of desmoid tumors. J Clin Oncol United States. 2007;25:1785-91.

3. Spear MA, Jennings LC, Mankin HJ, Spiro IJ, Springfield DS, Gebhardt MC, et al. Individualizing management of aggressive fibromatoses. Int J Radiat Oncol Biol Phys United States. 1998;40:63745.

4. Leithner A, Schnack B, Katterschafka T, Wiltschke C, Amann G, Windhager R, et al. Treatment of extraabdominal desmoid tumors with interferon-alpha with or without tretinoin. J Surg Oncol United States. 2000;73:21-5.

5. de Camargo VP, Keohan ML, D'Adamo DR, Antonescu CR, Brennan MF, Singer S, et al. Clinical outcomes of systemic therapy for patients with deep fibromatosis (desmoid tumor). Cancer United States. 2010;116:2258-65.

6. Chao AS, Lai CH, Hsueh S, Chen CS, Yang YC, Soong YK. Successful treatment of recurrent pelvic desmoid tumour with tamoxifen: case report. Hum Reprod England. 2000;15:311-3.

7. Azzarelli A, Gronchi A, Bertulli R, Tesoro JD, Baratti D, Pennacchioli E, et al. Low-dose chemotherapy with methotrexate and vinblastine for patients with advanced aggressive fibromatosis. Cancer United States. 2001;92:1259-64.

8. Penel N, Chibon F, Salas S. Adult desmoid tumors: biology, management and ongoing trials. Curr Opin Oncol United States. 2017;29:268-74.

9. Gounder MM, Mahoney MR, Van Tine BA, Ravi V, Attia S, Deshpande HA, et al. Sorafenib for Advanced and Refractory Desmoid Tumors. N Engl J Med [Internet]. Massachusetts Medical Society; 2018;379:2417-28. Available from: https://doi.org/10.1056/NEJMoa1805052.

10. Heinrich MC, McArthur GA, Demetri GD, Joensuu H, Bono P, Herrmann R, et al. Clinical and molecular studies of the effect of imatinib on advanced aggressive fibromatosis (desmoid tumor). J Clin Oncol United States. 2006;24:1195-203.

11. Chugh R, Wathen JK, Patel SR, Maki RG, Meyers PA, Schuetze SM, et al. Efficacy of imatinib in aggressive fibromatosis: Results of a phase II multicenter Sarcoma Alliance for Research through Collaboration (SARC) trial. Clin Cancer Res United States. 2010;16:4884-91.

12. Penel N, Le Cesne A, Bui BN, Perol D, Brain EG, Ray-Coquard I, et al. Imatinib for progressive and recurrent aggressive fibromatosis (desmoid tumors): an FNCLCC/French Sarcoma Group phase II trial with a long-term follow-up. Ann Oncol Off J Eur Soc Med Oncol England. 2011;22:452-7. 
13. Apperley JF, Gardembas M, Melo JV, Russell-Jones R, Bain BJ, Baxter EJ, et al. Response to imatinib mesylate in patients with chronic myeloproliferative diseases with rearrangements of the plateletderived growth factor receptor beta. N Engl J Med United States. 2002;347:481-7.

14. Demetri GD, von Mehren M, Blanke CD, Van den Abbeele AD, Eisenberg B, Roberts PJ, et al. Efficacy and safety of imatinib mesylate in advanced gastrointestinal stromal tumors. $\mathrm{N}$ Engl $\mathrm{J}$ Med United States. 2002;347:472-80.

15. Druker BJ, Talpaz M, Resta DJ, Peng B, Buchdunger E, Ford JM, et al. Efficacy and safety of a specific inhibitor of the BCR-ABL tyrosine kinase in chronic myeloid leukemia. N Engl J Med United States. 2001;344:1031-7.

16. Eisenhauer EA, Therasse P, Bogaerts J, Schwartz LH, Sargent D, Ford R, et al. New response evaluation criteria in solid tumours: revised RECIST guideline (version 1.1). Eur J Cancer England. 2009;45:228-47.

17. McKenna A, Hanna M, Banks E, Sivachenko A, Cibulskis K, Kernytsky A, et al. The Genome Analysis Toolkit: a MapReduce framework for analyzing next-generation DNA sequencing data. Genome Res [Internet]. 2010 [cited 2014 May 25];20:1297-303. Available from:

http://www.pubmedcentral.nih.gov/articlerender.fcgi? artid $=2928508 \&$ tool $=$ pmcentrez\&rendertype $=$ abstract.

18. Li H, Durbin R. Fast and accurate short read alignment with Burrows-Wheeler transform. Bioinformatics [Internet]. 2009;25:1754-60. Available from: http://www.pubmedcentral.nih.gov/articlerender.fcgi? artid $=2705234 \&$ tool $=$ pmcentrez\&rendertype $=$ abstract.

19. Cibulskis K, Lawrence MS, Carter SL, Sivachenko A, Jaffe D, Sougnez C, et al. Sensitive detection of somatic point mutations in impure and heterogeneous cancer samples. Nat Biotechnol [Internet]. Nature Publishing Group; 2013 [cited 2018 Aug 5];31:213-9. Available from: http://www.nature.com/articles/nbt.2514.

20. Yang H, Wang K. Genomic variant annotation and prioritization with ANNOVAR and wANNOVAR. Nat Protoc. Nature Publishing Group, a division of Macmillan Publishers Limited. All Rights Reserved. 2015;10:1556.

21. Thorvaldsdottir H, Robinson JT, Mesirov JP. Integrative Genomics Viewer (IGV): high-performance genomics data visualization and exploration. Brief Bioinform England. 2013;14:178-92.

22. Lek M, Karczewski KJ, Minikel EV, Samocha KE, Banks E, Fennell T, et al. Analysis of protein-coding genetic variation in 60,706 humans. Nature. 2016;536:285. The Author(s).

23. Kim K, Jang K, Yang W, Choi E-Y, Park S-M, Bae M, et al. Chromatin structure-based prediction of recurrent noncoding mutations in cancer. Nat Genet. 2016.

24. Vogelstein B, Papadopoulos N, Velculescu VE, Zhou S Jr LAD, Kinzler KW. Cancer genome landscapes. Science. 2013;339:1546-58.

25. Sanyal A, Lajoie BR, Jain G, Dekker J. The long-range interaction landscape of gene promoters. Nature. 2012;489:109-13. 
26. Harrow J, Frankish A, Gonzalez JM, Tapanari E, Diekhans M, Kokocinski F, et al. GENCODE: The reference human genome annotation for the ENCODE project. Genome Res. 2012;22:1760-74.

27. Kircher M, Witten DM, Jain P, O'Roak BJ, Cooper GM, Shendure J. A general framework for estimating the relative pathogenicity of human genetic variants. Nat Genet [Internet]. Nature Research; 2014 [cited 2016 Aug 6];46:310-5. Available from: http://www.nature.com/doifinder/10.1038/ng.2892.

28. Quang $D, C h e n Y, X i e X$. DANN: a deep learning approach for annotating the pathogenicity of genetic variants. Bioinformatics [Internet]. 2015;31:761-3. Available from:

http://dx.doi.org/10.1093/bioinformatics/btu703.

29. Campbell PJ, Getz G, Korbel JO, Stuart JM, Jennings JL, Stein LD, et al. Pan-cancer analysis of whole genomes. Nature. 2020;578.

30. Martin M. Cutadapt removes adapter sequences from high-throughput sequencing reads. EMBnet $J$ [Internet]. 2011;17:10. Available from: https://app.dimensions.ai/details/publication/pub.1067372670.

31. Dobin A, Davis CA, Schlesinger F, Drenkow J, Zaleski C, Jha S, et al. STAR: ultrafast universal RNAseq aligner. Bioinformatics. 2013;29:15-21.

32. Li B, Dewey CN. RSEM: accurate transcript quantification from RNA-Seq data with or without a reference genome. 2011.

33. Wang L, Wang S, Li W. RSeQC: Quality control of RNA-seq experiments. Bioinformatics. 2012;28:2184-5.

34. Ewels P, Magnusson M, Lundin S, Käller M. MultiQC: summarize analysis results for multiple tools and samples in a single report. Bioinformatics [Internet]. 2016;32:3047-8. Available from: https://doi.org/10.1093/bioinformatics/btw354.

35. Kuleshov MV, Jones MR, Rouillard AD, Fernandez NF, Duan Q, Wang Z, et al. Enrichr: a comprehensive gene set enrichment analysis web server 2016 update. Nucleic Acids Res. Oxford Univ Press; 2016. gkw377.

36. Subramanian A, Tamayo P, Mootha VK, Mukherjee S, Ebert BL, Gillette MA, et al. Gene set enrichment analysis: A knowledge-based approach for interpreting genome-wide expression profiles. Proc Natl Acad Sci U S A; 2005.

37. Rosenthal R, McGranahan N, Herrero J, Taylor BS, Swanton C. deconstructSigs: delineating mutational processes in single tumors distinguishes DNA repair deficiencies and patterns of carcinoma evolution. Genome Biol. 2016;17:31.

38. Forbes SA, Beare D, Boutselakis H, Bamford S, Bindal N, Tate J, et al. COSMIC: somatic cancer genetics at high-resolution. Nucleic Acids Res [Internet]. 2017;45:D777-83. Available from: http://dx.doi.org/10.1093/nar/gkw1121.

39. Le Guellec S, Soubeyran I, Rochaix P, Filleron T, Neuville A, Hostein I, et al. CTNNB1 mutation analysis is a useful tool for the diagnosis of desmoid tumors: a study of 260 desmoid tumors and 191 potential morphologic mimics. Mod Pathol [Internet]. United States and Canadian Academy of Pathology, Inc.; 2012;25:1551. Available from: http://dx.doi.org/10.1038/modpathol.2012.115. 
40. Mullen JT, DeLaney TF, Rosenberg AE, Le L, lafrate AJ, Kobayashi W, et al. beta-Catenin mutation status and outcomes in sporadic desmoid tumors. Oncologist United States. 2013;18:1043-9.

41. Lazar AJF, Tuvin D, Hajibashi S, Habeeb S, Bolshakov S, Mayordomo-Aranda E, et al. Specific mutations in the beta-catenin gene (CTNNB1) correlate with local recurrence in sporadic desmoid tumors. Am J Pathol United States. 2008;173:1518-27.

42. Liu Z-H, Dai X-M, Du B. Hes1: a key role in stemness, metastasis and multidrug resistance. Cancer Biol Ther United States. 2015;16:353-9.

43. Carothers AM, Rizvi H, Hasson RM, Heit YI, Davids JS, Bertagnolli MM, et al. Mesenchymal stromal cell mutations and wound healing contribute to the etiology of desmoid tumors. Cancer Res United States. 2012;72:346-55.

44. Eskandari S, Yazdanparast R. Overexpression of Hes1 is involved in sensitization of K562 cells to Imatinib. J Cell Biochem United States. 2019;120:10128-36.

45. Bouitbir J, Panajatovic MV, Frechard T, Roos NJ, Krähenbühl S. Imatinib and Dasatinib Provoke Mitochondrial Dysfunction Leading to Oxidative Stress in C2C12 Myotubes and Human RD Cells. Front Pharmacol. 2020;11:1106.

46. Shyh-Chang N, Ng H-H. The metabolic programming of stem cells. Genes Dev. 2017;31:336-46.

47. Watt SM, Gullo F, van der Garde M, Markeson D, Camicia R, Khoo CP, et al. The angiogenic properties of mesenchymal stem/stromal cells and their therapeutic potential. Br Med Bull. 2013;108:25-53.

48. Escobar C, Munker R, Thomas JO, Li BD, Burton GV. Update on desmoid tumors. Ann Oncol Off J Eur Soc Med Oncol England. 2012;23:562-9.

49. Messersmith WA, Shapiro GI, Cleary JM, Jimeno A, Dasari A, Huang B, et al. A Phase I, Dose-Finding Study in Patients with Advanced Solid Malignancies of the Oral Y-Secretase Inhibitor PF-03084014. Clin Cancer Res [Internet]. 2015;21:60 LP - 67. Available from: http://clincancerres.aacrjournals.org/content/21/1/60.abstract.

50. Timbergen MJM, Smits R, Grünhagen DJ, Verhoef C, Sleijfer S, Wiemer EAC. Activated Signaling Pathways and Targeted Therapies in Desmoid-Type Fibromatosis: A Literature Review [Internet]. Front. Oncol.. 2019. p. 397. Available from: https://www.frontiersin.org/article/10.3389/fonc.2019.00397.

51. Kobayashi T, Terada Y, Kuwana H, Tanaka H, Okado T, Kuwahara M, et al. Expression and function of the Delta-1/Notch-2/Hes-1 pathway during experimental acute kidney injury. Kidney Int United States. 2008;73:1240-50.

52. Hayashi Y, Osanai M, Lee G-H NOTCH2 signaling confers immature morphology and aggressiveness in human hepatocellular carcinoma cells. Oncol Rep [Internet]. Department of Pathology, Kochi University School of Medicine, Kohasu, Oko-cho, Nankoku, Kochi 783-8505, Japan; 2015;34:1650-8. Available from: https://doi.org/10.3892/or.2015.4171.

53. Aljedai A, Buckle A-M, Hiwarkar P, Syed F. Potential Role of Notch Signalling in CD34+ Chronic Myeloid Leukaemia Cells: Cross-Talk between Notch and BCR-ABL. PLoS One [Internet]. Public 
Library of Science; 2015;10:e0123016. Available from:

https://doi.org/10.1371/journal.pone.0123016.

54. Callahan R, Chestnut BA, Raafat A. Original Research: Featured Article: Imatinib mesylate (Gleevec) inhibits Notch and c-Myc signaling: Five-day treatment permanently rescues mammary development. Exp Biol Med (Maywood) England. 2017;242:53-67.

55. Netzer WJ, Dou F, Cai D, Veach D, Jean S, Li Y, et al. Gleevec inhibits beta-amyloid production but not Notch cleavage. Proc Natl Acad Sci U S A United States. 2003;100:12444-9.

56. Weggen S, Eriksen JL, Das P, Sagi SA, Wang R, Pietrzik CU, et al. A subset of NSAIDs lower amyloidogenic Abeta42 independently of cyclooxygenase activity. Nature England. 2001;414:212-6.

57. Kim H-A, Koo B-K, Cho J-H, Kim Y-Y, Seong J, Chang HJ, et al. Notch1 counteracts WNT/beta-catenin signaling through chromatin modification in colorectal cancer. J Clin Invest United States. 2012;122:3248-59.

58. Benedito R, Hellstrom M. Notch as a hub for signaling in angiogenesis. Exp Cell Res United States. 2013;319:1281-8.

59. Batlle E, Clevers H. Cancer stem cells revisited. Nat Med United States. 2017;23:1124-34.

60. Meurette O, Mehlen P. Notch Signaling in the Tumor Microenvironment. Cancer Cell. 2018.

61. Espinosa L, Ingles-Esteve J, Aguilera C, Bigas A. Phosphorylation by glycogen synthase kinase-3 beta down-regulates Notch activity, a link for Notch and Wnt pathways. J Biol Chem United States. 2003;278:32227-35.

62. Shang H, Braggio D, Lee Y-J, Al Sannaa GA, Creighton CJ, Bolshakov S, et al. Targeting the Notch pathway: A potential therapeutic approach for desmoid tumors. Cancer United States. 2015;121:4088-96.

63. Kummar S, O'Sullivan Coyne G, Do KT, Turkbey B, Meltzer PS, Polley E, et al. Clinical Activity of the gamma-Secretase Inhibitor PF-03084014 in Adults With Desmoid Tumors (Aggressive Fibromatosis). J Clin Oncol United States. 2017;35:1561-9.

64. Arcaroli JJ, Quackenbush KS, Purkey A, Powell RW, Pitts TM, Bagby S, et al. Tumours with elevated levels of the Notch and Wnt pathways exhibit efficacy to PF-03084014, a gamma-secretase inhibitor, in a preclinical colorectal explant model. Br J Cancer England. 2013;109:667-75.

65. Ungerbäck J, Elander N, Grünberg J, Sigvardsson M, Söderkvist P. The Notch-2 Gene Is Regulated by Wnt Signaling in Cultured Colorectal Cancer Cells. PLoS One Public Library of Science. 2011;6:1-13.

\section{Figures}




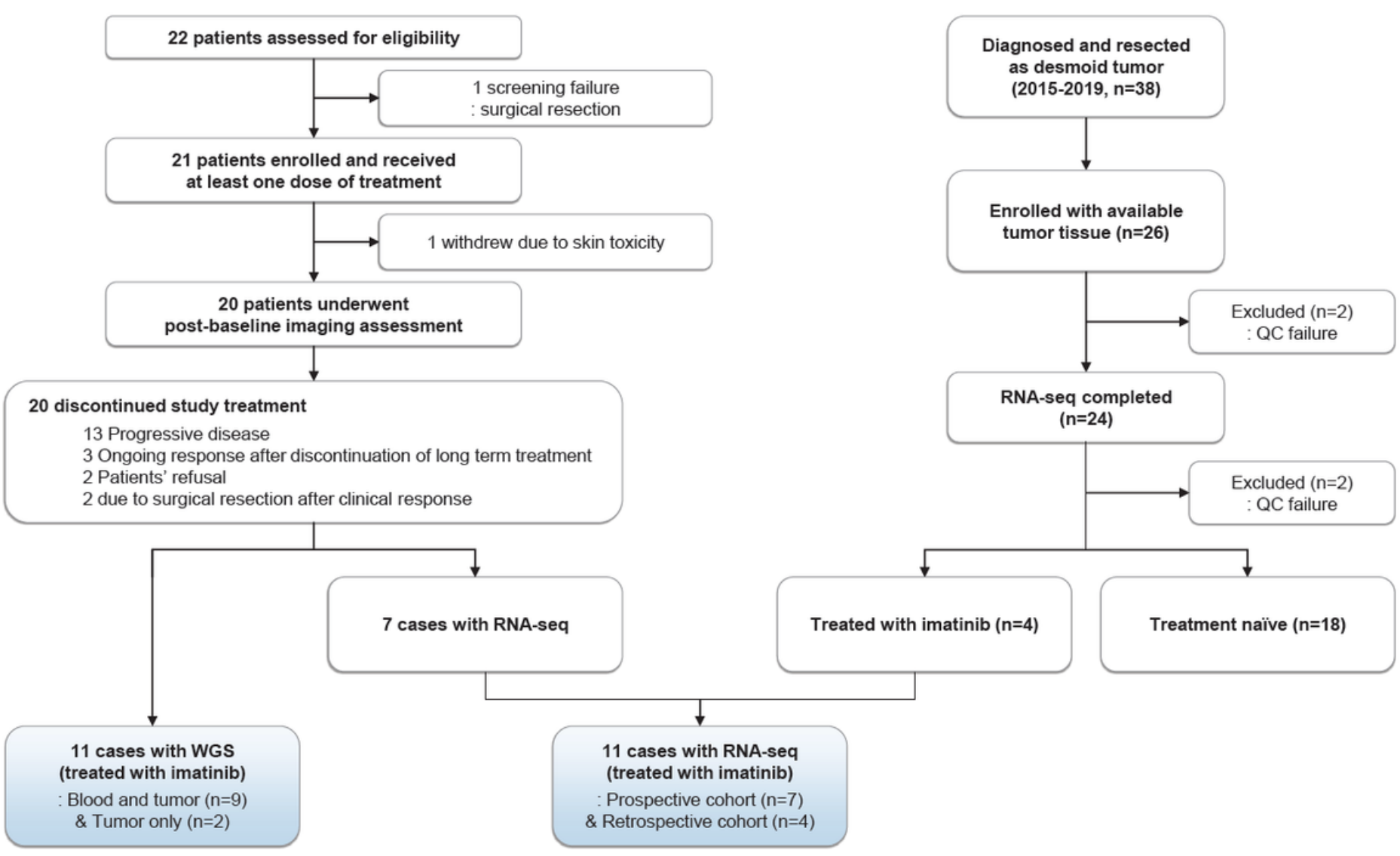

Figure 1

Consort diagram of desmoid tumor patients included in this study. Prospective phase II study with treatment of imatinib included 20 patients after excluding 2 patients with clinical concerns. Tumor samples from 11 patients, which passed stringent quality check, were whole-genome-sequenced. RNAseq was performed for 29 cases, including 7 in phase II study and 22 in retrospective cohort, of which 11 cases were treated with imatinib. 
A

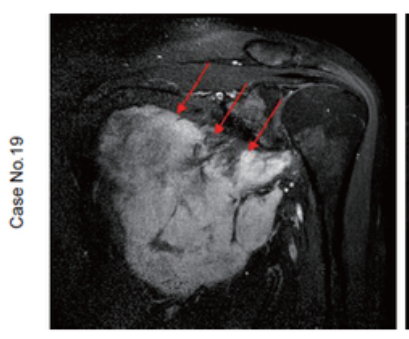

Baseline

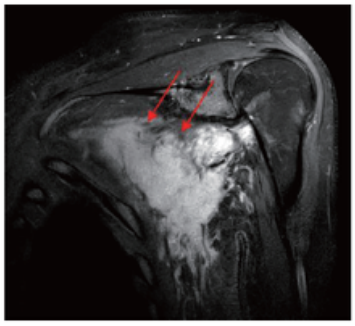

24 weeks after imitinib
B

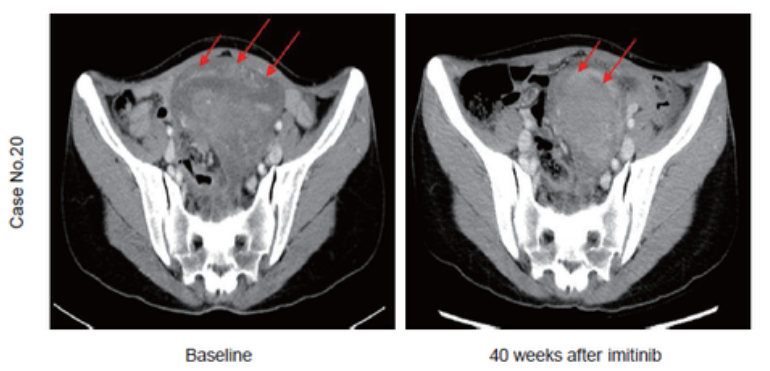

D

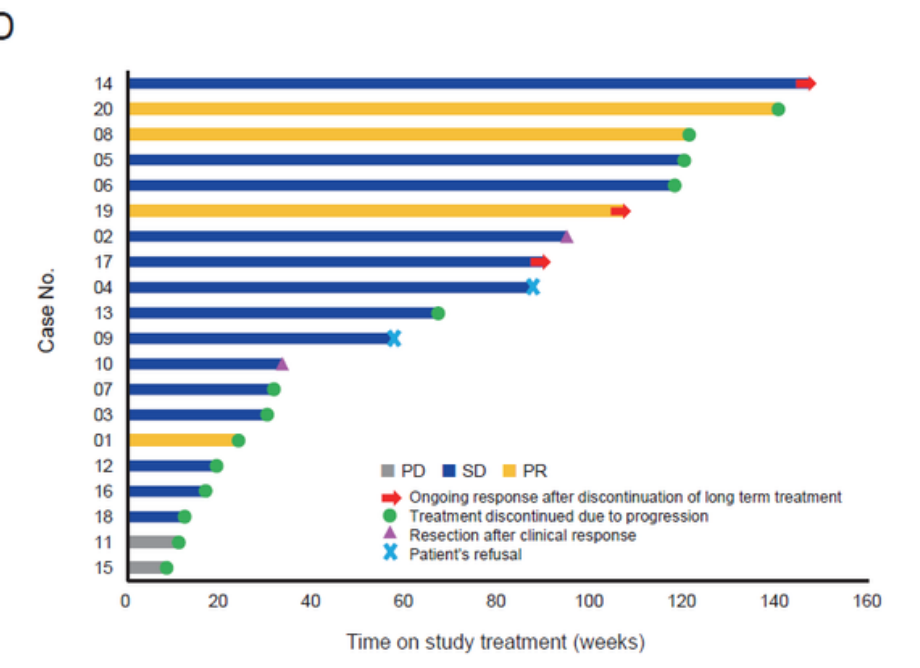

40 weeks after imitinib

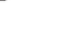

(1)
C

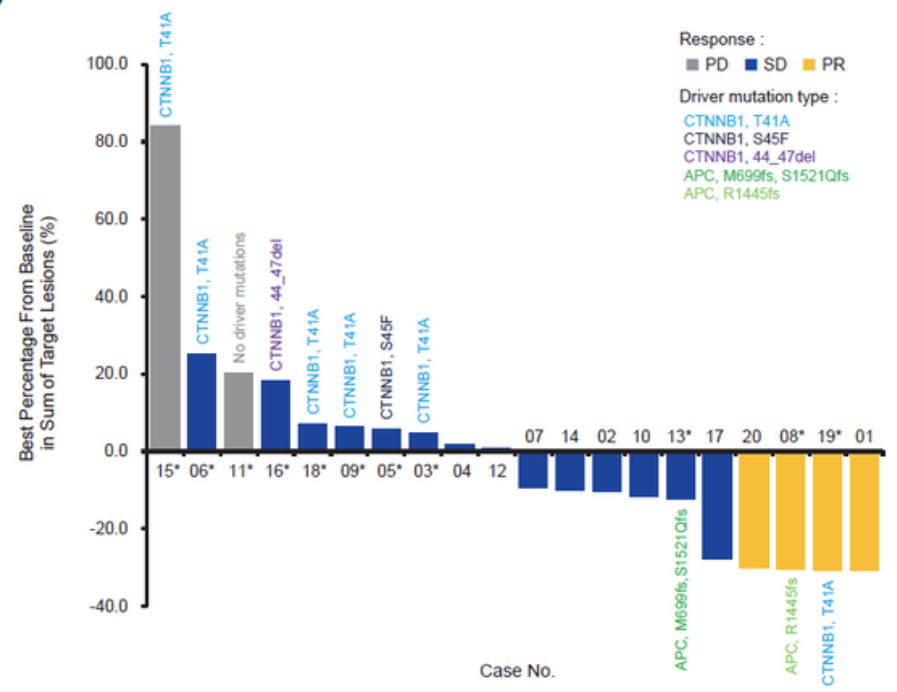

\section{Figure 2}

Representative pre- and post-treatment imaging scans to show antitumor activity of imatinib in desmoid tumors. Soft tissue lesion in the shoulder of patient \#19 (A) and pelvis of patient \#20 (B) exhibited a significant size reduction after 24 and 40 weeks of imatinib treatment (red arrow). C, Relative change in tumor volume of patients $(n=20)$ over time. * indicates a sample with WGS data. The label at the end of the bar shows mutational information in known driver genes, CTNNB1 and APC, for desmoid tumor. D, Swimmer plot. Each lane represents a single patient's data. $\mathrm{X}$ axis represents the duration of treatment for each patient. 
A
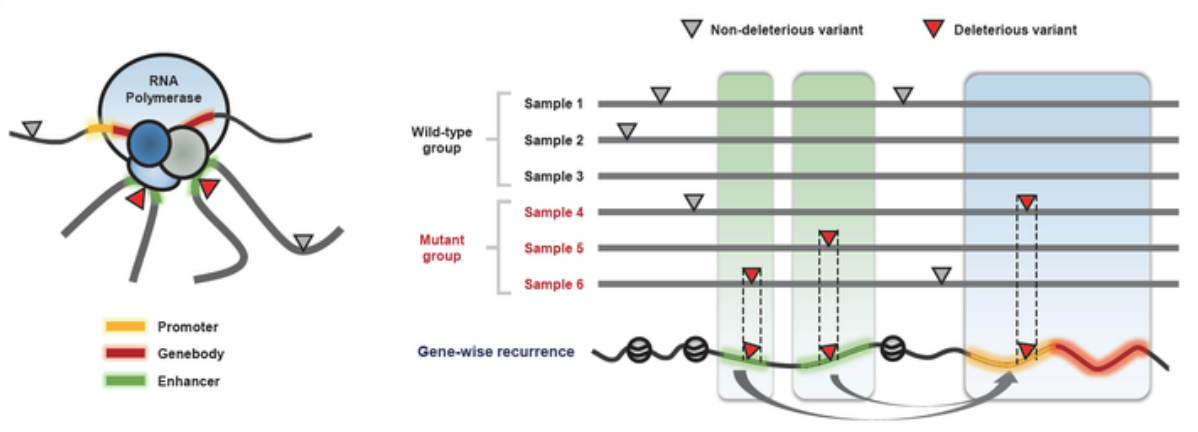

B

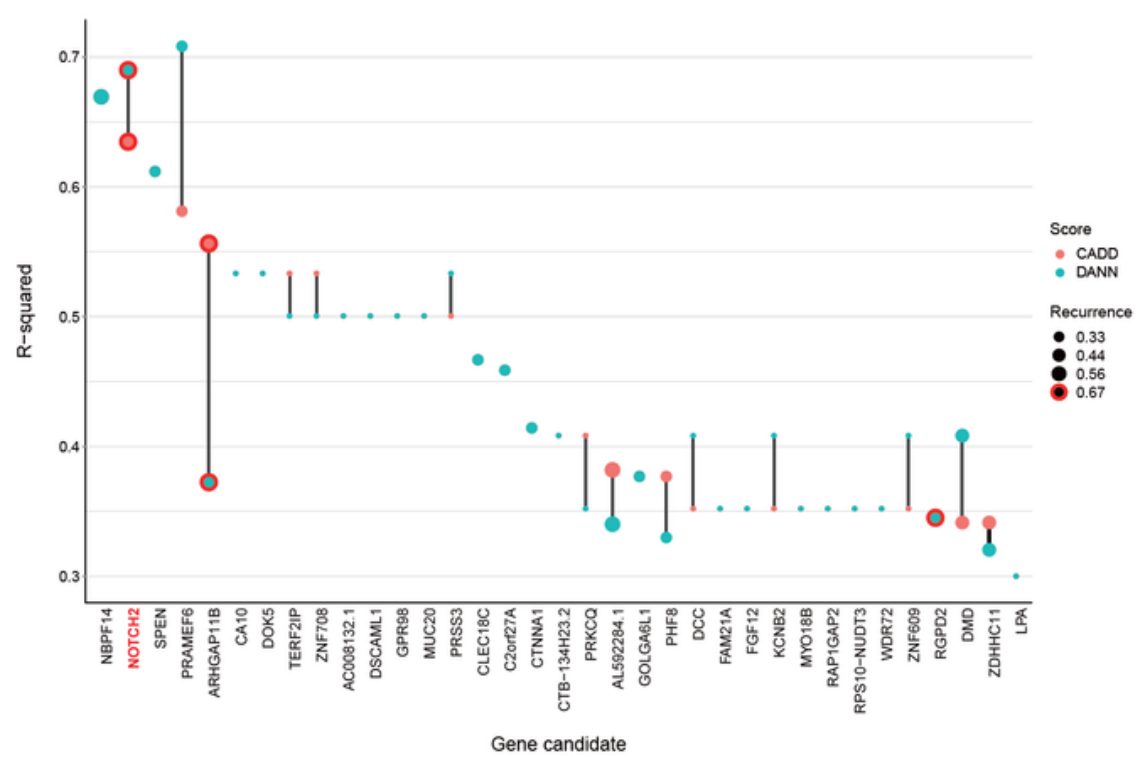

C

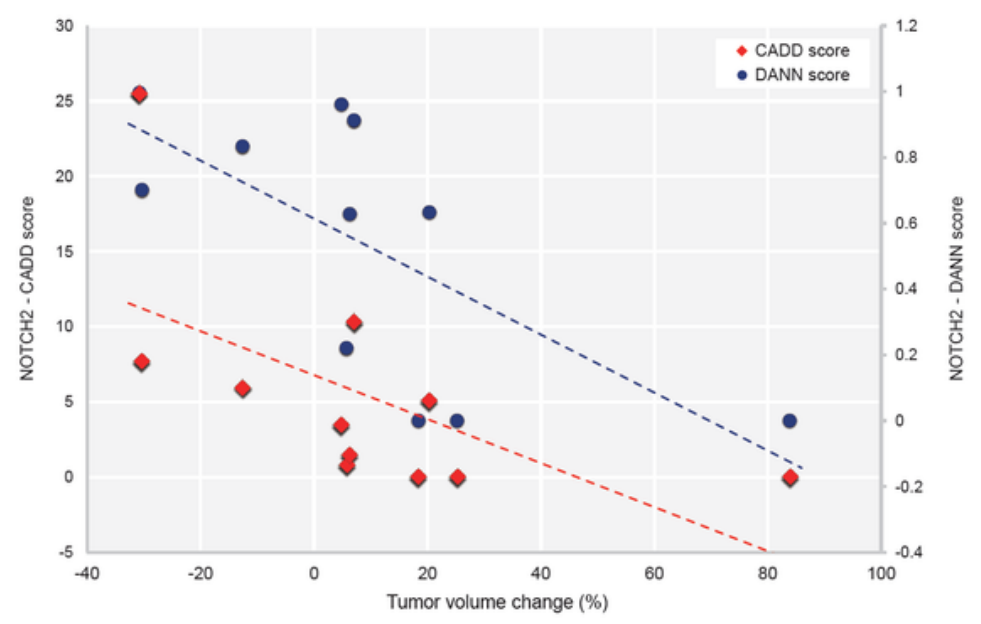

\section{Figure 3}

Gene-wise recurrence of noncoding mutations. A, Definition of functional noncoding mutations and schematic overview of the gene-wise recurrence model. Functional noncoding mutations in regulatory regions, such as enhancers, affect the expression level of the target gene. Mutations outside of functional regions were excluded from our analysis. Functional mutations were identified as recurrent if they occur in regulatory regions converging of the same gene via enhancer-promoter chromatin interactions across 
multiple patients. Deleteriousness of functional mutations in mutant groups were quantified using CADD and DANN. B, Cleveland plot shows correlation coefficients (R2) for the relationship between tumor volume change and deleteriousness score, and recurrence for each gene in desmoid tumor patients $(n=9)$. Genes are ordered by the magnitude of R-squared value. Only protein-coding genes with a recurrence value of 2 or greater are shown. $\mathrm{C}, \mathrm{CADD}$ scores for $\mathrm{NOTCH} 2$ mutations according to tumor volume changes were plotted with the regression line shown in red $(R=-0.716, P=0.013)$. DANN scores for NOTCH2 mutations were also plotted with the regression line shown in blue $(R=-0.83, P=0.006)$. Each point represents a patient's sample. 
A

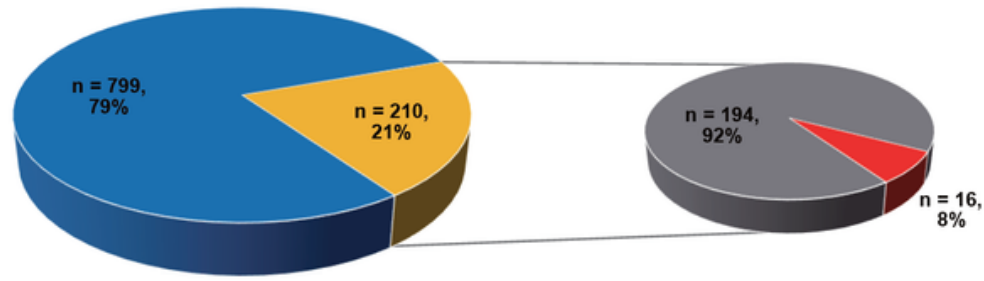

without-NOTCH2 mut

only-NOTCH2 mut with-NOTCH2 mut

both-NOTCH2-CTNNB1 mut

B

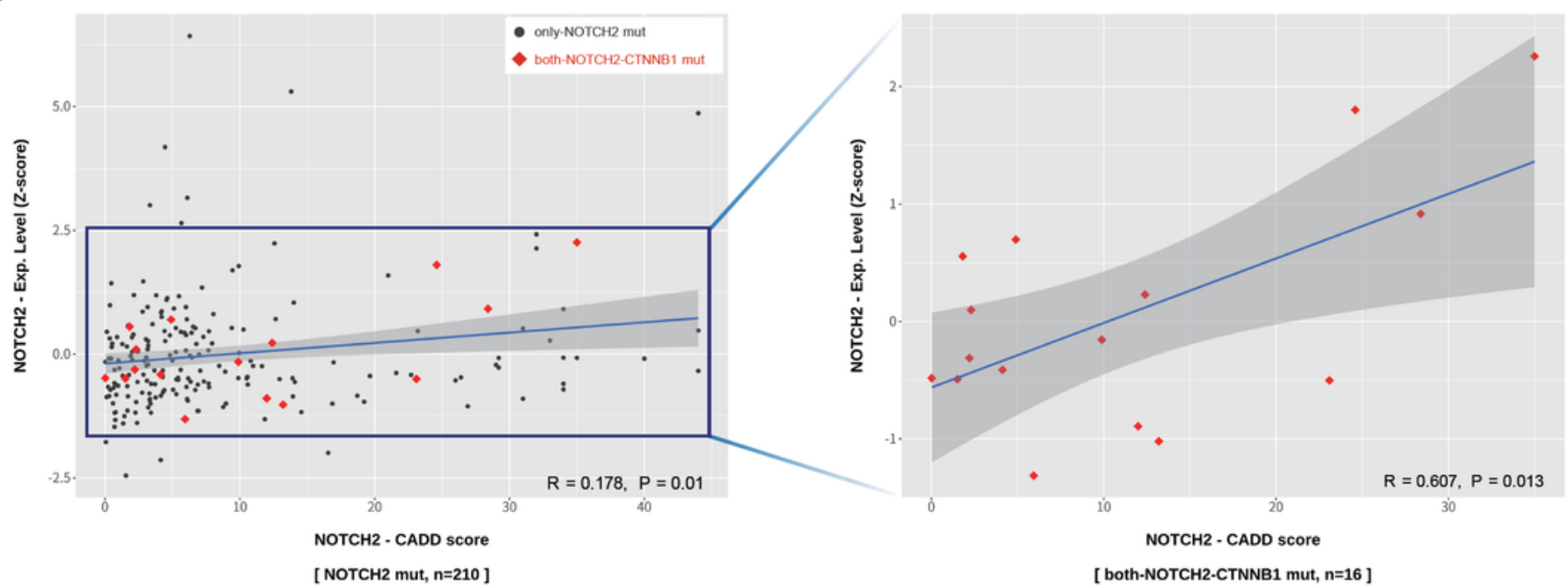

C

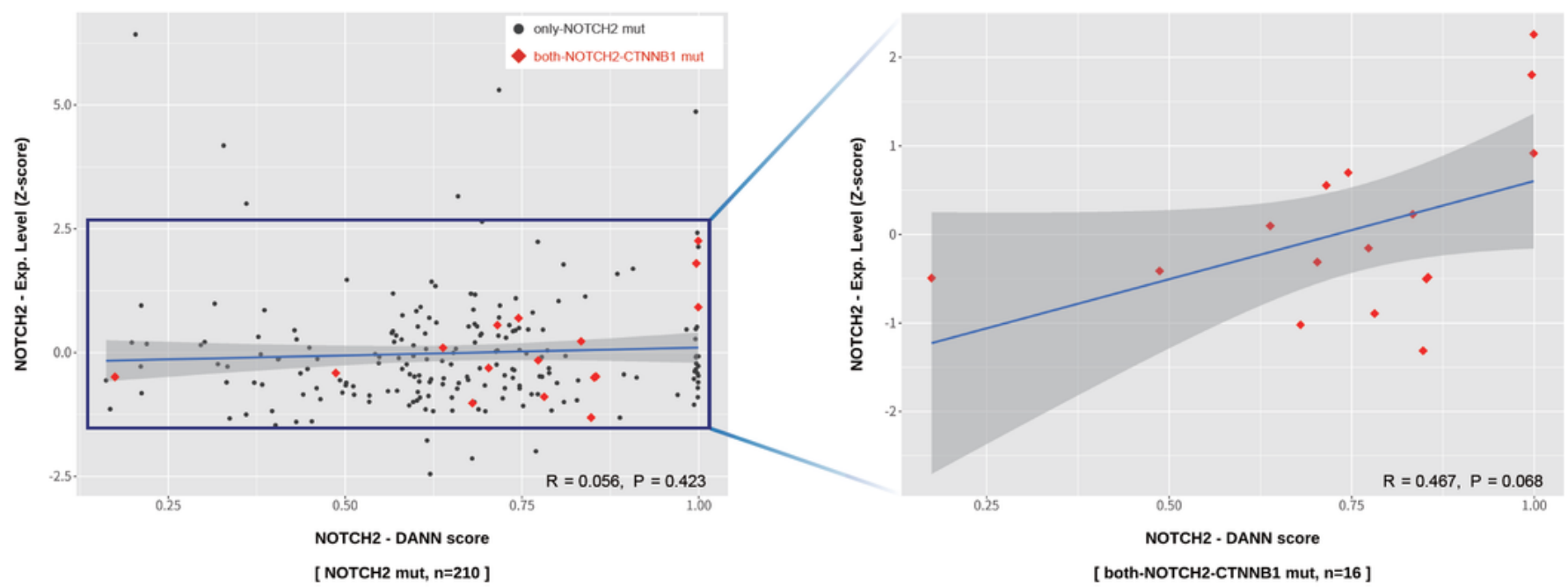

Figure 4

Correlation between the deleteriousness of NOTCH2 noncoding-mutations and expression level of NOTCH 2 in PCAWG cohort. A, Proportions of patients with NOTCH2 mutations $(n=210)$ and with both NOTCH2 and CTNNB1 missense mutations $(n=16)$. B, Correlation between CADD score and NOTCH2 expression level in the group with $\mathrm{NOTCH} 2$ mutations (left side) and in selected samples harboring both NOTCH2 and CTNNB1 missense mutations (right side). C, The correlation analysis was repeated using 
DANN score in the group with NOTCH2 mutations (left side) and in selected samples with both NOTCH2 and CTNNB1 missense mutations (right side).

A
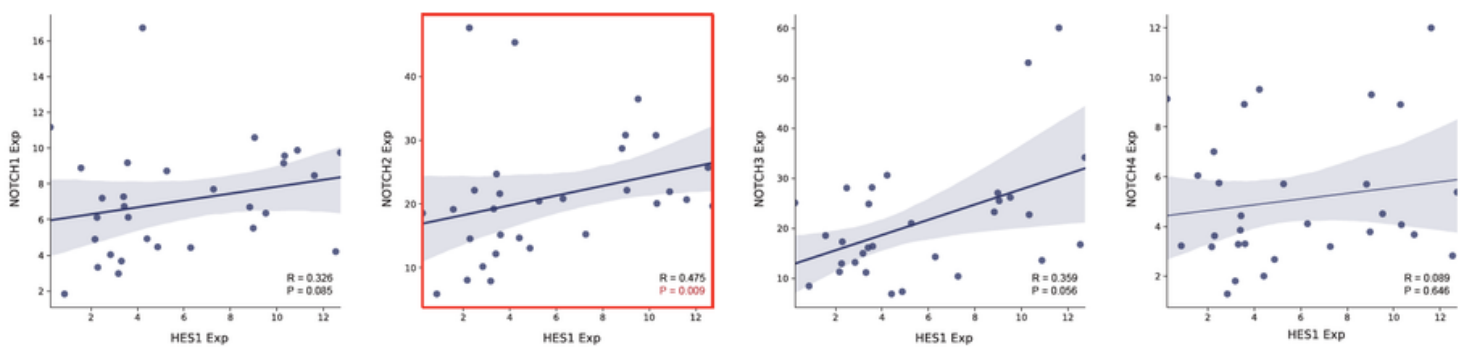

B
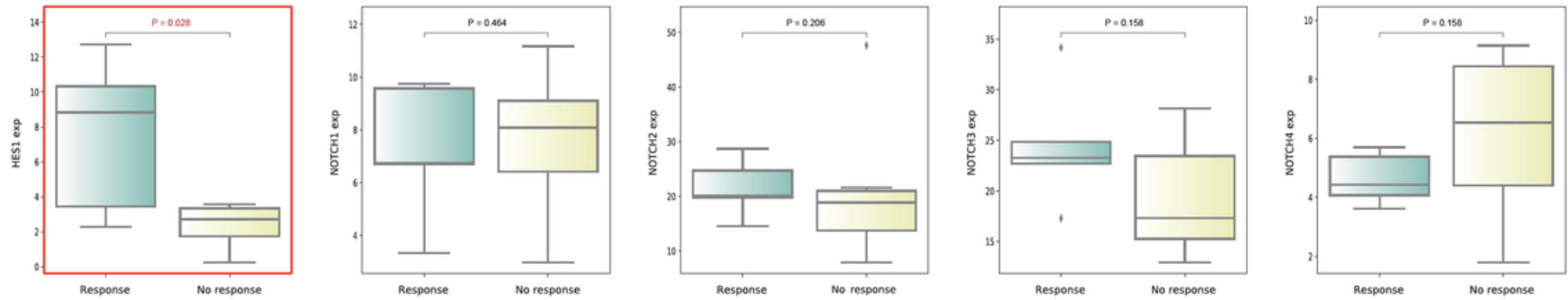

C
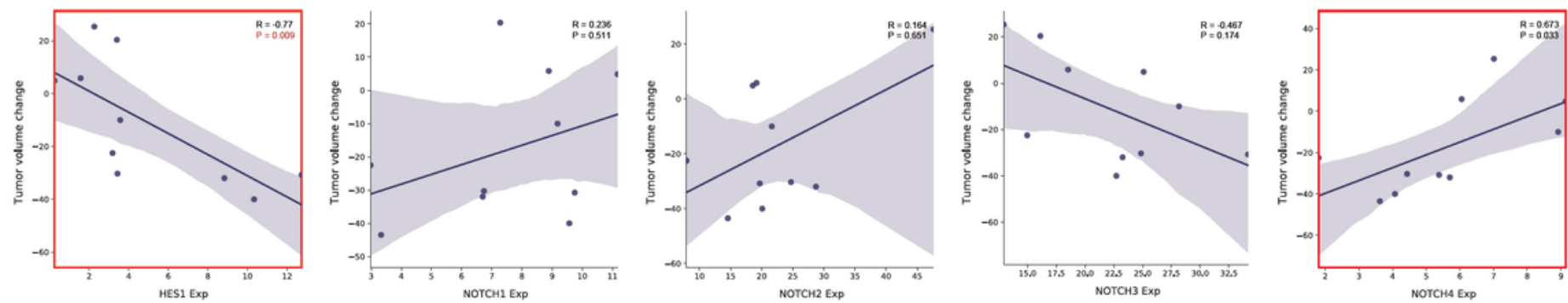

\section{Figure 5}

Expressional association between Notch family and HES1 genes, and its clinical significance in desmoid tumor samples $(n=29)$. A, Co-expression between HES1 and one of Notch family genes, including $\mathrm{NOTCH} 1, \mathrm{NOTCH} 2, \mathrm{NOTCH} 3$ and NOTH4, respectively. Correlation analysis was calculated using spearman and all expression levels are in TPM values. B, Expressional differences of HES1, NOTCH1, NOTCH2, NOTCH3 and NOTCH4, between responders $(n=5)$ and non-responders $(n=6)$ after treatment of imatinib. Statistical significance was determined using Mann-Whitney U test. C, Correlation between tumor volume change, indicating the imatinib-response, and gene expression level: HES1 and Notch family members. Plots depict correlation analysis after removing one outlier. All red square lines indicate statistical significance $(P<0.05)$. 
A

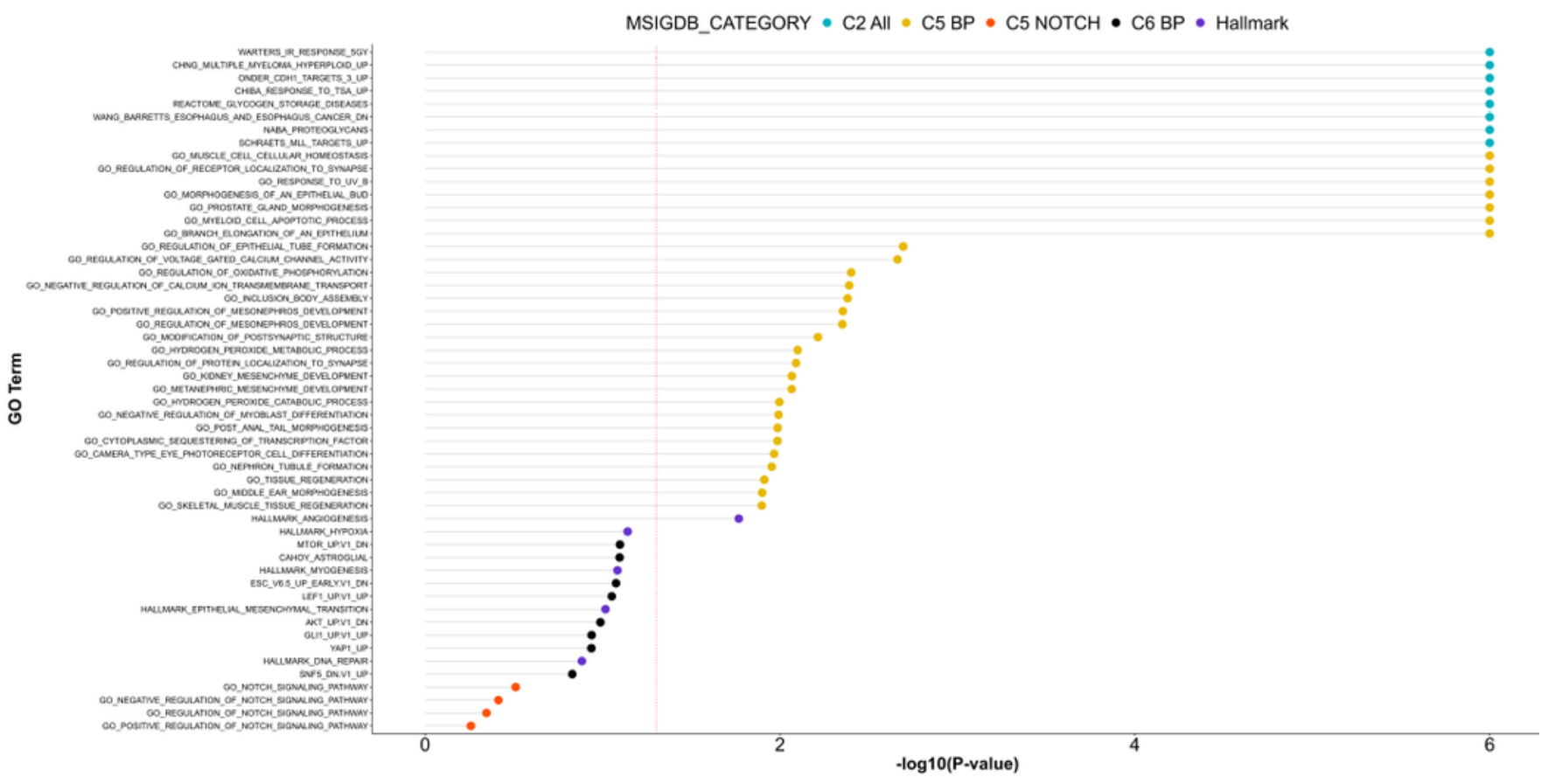

B

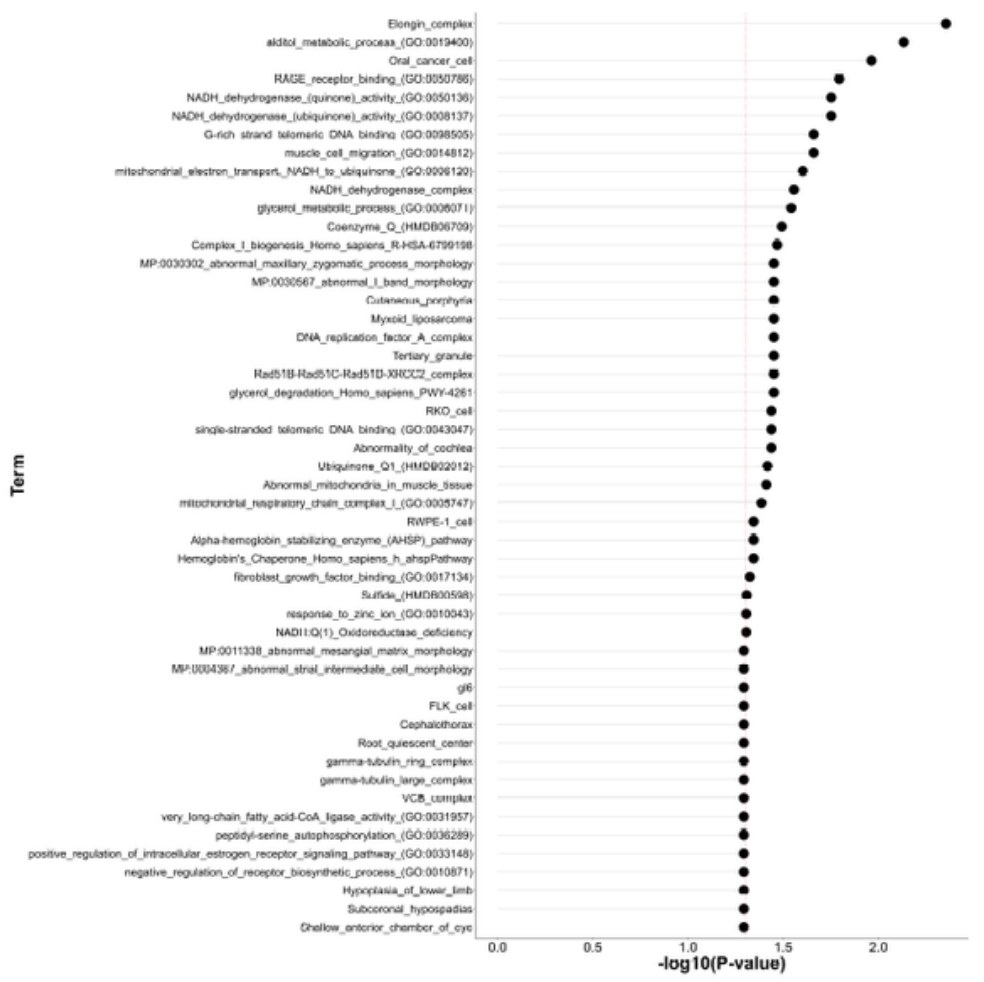

Figure 6

A, GSEA for differential genes with statistical importance $(P<0.05)$ between responders $(n=5)$ and nonresponders $(n=6)$. GO terms on y-axis indicate molecular signatures database (MSigDB), of which five gene sets are shown in legend: C2, as curated gene sets; C5 BP, as gene sets from biological process ontology; C5 NOTCH, as gene sets associated with Notch pathway; C6 BP, as oncogenic signatures of biological process; Hallmark, as gene sets to represent specific biological states or processes and show 
consistent expression. Most significant terms for each category are depicted. B, GSEA result by using EnrichR with genes that are significantly correlated with imatinib sensitivity. Red vertical lines indicate $p$ value of 0.05 .

\section{Supplementary Files}

This is a list of supplementary files associated with this preprint. Click to download.

- Additionalfile1Tables1.pdf

- Additionalfile2Tables2.pdf

- Additionalfile3Tables3.pdf

- Additionalfile4Tables4.pdf

- Additionalfile5Tables5.pdf

- Additionalfile6Figures1.pdf

- Additionalfile7Tables6.pdf

- Additionalfile8Tables7.pdf

- Additionalfile9Tables8.pdf

- Additionalfile10TableS9.pdf

- Additionalfile11FigureS2.pdf

- SupplementaryMaterialProtocollmatinibDesmoidTumor.docx 